\title{
Südafrika zwischen Vormacht und Ohnmacht
}

\author{
Von Klaus Frhr. von der Ropp
}

\section{Pax Americana versus Pax Pretoriana}

Bekanntlich wurde den Problemen des südlichen Afrikas, und hier insbesondere jenen der Republik Südafrika, während der Amtszeit des Präsidenten Jimmy Carter in Washington hohe Priorität eingeräumt. Er war, geprägt durch die Bürgerrechtsbewegung in den USA, auch persönlich an der Uberwindung der Apartheid zugunsten der Ubernahme des, wie seine führenden Mitarbeiter dies formulierten, "amerikanischen Modells" durch Südafrika interessiert. So wurde berichtet, Carter habe geäußert, daß für den Fall, daß er nicht zum amerikanischen Regierungschef gewählt worden wäre, er gerne als Bürgerrechtskämpfer nach Südafrika gegangen wäre. Carter und jene nicht wenigen westeuropäischen Politiker, die seine Sicht der Probleme Südafrikas teilten, scheiterten mit ihrer Politik daran, daß sie in einer dem Sachkundigen nicht nachvollziehbaren Weise den weißafrikanischen Widerstand gegen eine Herrschaftsumkehr in Windhoek und Pretoria unterschätzten und sich daher entsprechend wenig Gedanken zu dessen notfalls gewaltsamer Uberwindung machten. ${ }^{1}$

Ermutigt durch diese Erfahrung war Pretoria in den folgenden Jahren mit gutem Erfolg bemüht, auch die durch den sehr sachkundigen Chester A. Crocker formulierte Politik der Regierung Ronald Reagan zum Scheitern zu bringen. Sie war und ist auf einen fairen Interessenausgleich ausgerichtet, in den Augen Pretorias war sie gleichwohl verfehlt. Denn, so eine in Südafrika verbreitete Auffassung, so unterschätzt die vom "Weltkommunismus" ausgehenden Gefahren. So widersetzen sich die "anti-kommunistischen" Gralshüter in Pretoria den Vorstellungen Reagans kaum weniger als zuvor jenen Carters, die sie mit Recht als naiv abgetan hatten. Deutlich wurde die Haltung u. a. in dem Vortrag, den der damalige Ministerpräsident P. W. Botha Anfang Juni 1984 gelegentlich eines Aufenthaltes in der Bundesrepublik Deutschland vor den Mitgliedern der Deutschen Gesellschaft für Auswärtige Politik in Bonn hielt. ${ }^{2}$ Es wurde die Erwartung

1 Kritisch zu der Politik der Carter Administration Klaus Frhr. von der Ropp "Konfliktherde im südlichen Afrika - Rhodesien, Namibia, Südafrika" in "Die Internationale Politik 1977/78«, S. 269-285, Jahrbuch der DGAP, Bonn, 1982; ders. "Südafrika und Namibia: Das Scheitern der Friedensinitiativen", S. 338-357 in "Die Internationale Politik 1979/80", Jahrbuch der DGAP, Bonn, 1983. Zu einer positiven Einschätzung dieser Politik kommt hingegen Winrich Kühne "Südafrika und seine Nachbarn: Durchbruch zum Frieden?" Baden-Baden, 1985, insbesondere S. 113-122.

2 Der Vortrag ist abgedruckt in South Africa International (Johannesburg) 1984. Oktober, S. 66-72 ("Peace Initiatives in Southern Africa"). 
deutlich, das südliche Afrika und dann auch die übrige Welt werde es lernen, mit der Politiker der Getrennten Entwicklung (d. i. Apartheid) zu leben. Hier sprach der Architekt der Pax Pretoriana. ${ }^{3}$

Unter dem Eindruck der Unfähigkeit Pretorias, nach Innen und nach Außen eine Politik des Friedens zumindest zu suchen, ist die Politik Washingtons inzwischen härter geworden. ${ }^{4}$ In den USA sieht man deutlich die Gefahr, daß die Politik der weißen Afrikaner den Subkontinent in einen einzigen Abgrund der Gewalt stürzen wird. Wichtig ist, daß man trotz der Kritik an dem bisherigen Kurs nicht auf die Linie der Carterschen Gemeinplätze zurückgefallen ist.

\section{Einige Aspekte der südaf rikanischen Regionalpolitik}

Bereits Ende der siebziger Jahre hatte Südafrika damit begonnen, unter konsequenter und gekonnter Ausnutzung von deren Instabilitäten und wirtschaftlichen Abhängigkeiten jene seiner Nachbarstaaten zu destabilisieren, die der Revolution in SWA/Namibia und Südafrika Vorschub leisteten.

Es war dann vor allem der Mittlerrolle der USA und von im Hintergrund gebliebenen Portugiesen zuzuschreiben, daß sich 1984 ein Rahmen friedensstiftender Verträge abzeichnete, innerhalb dessen, so eine damals verbreitete Hoffnung, sich, recht kurzfristig, der SWA/Namibia-Konflikt und, eher länger- als mittelfristig, dann auch die politischen und wirtschaftlichen Probleme Südafrikas würden lösen lassen.

Ein erster Durchbruch auf dem Weg der Uberwindung eskalierender Gewalt schien zu gelingen, als im Februar 1984 in Lusaka das angolanisch-südafrikanische Truppenentflechtungsabkommen unterzeichnet wurde. Infolge von dem Vertrag entgegenstehenden Aktivitäten der People's Liberation Army (PLAN) Swapos zogen die letzten südafrikanischen Truppen erst Mitte 1985 aus (Süd-)-Angola ab. Zum Abschluß eines Waffenstillstandes zwischen Pretoria und SWAPO kam es jedoch nicht. Entsprechende Bemühungen, die bei der SWA/Namibia-Konferenz im Mai 1984 in Lusaka und in bilateralen Verhandlungen zwischen Emissären der kriegführenden Parteien unternommen wurden, blieben erfolglos. Denn eine Einigung über das anschließende Procedere, nach dem SWA/Namibia unabhängig werden sollte, war nicht möglich. SWAPO bestand auf der

3 S. dazu Theodor Hanf "Konflikte im südlichen Afrika ", S. 651-662 in Karl Kaiser und Hans-Peter Schwarz (Hrsg.) "Weltpolitik/Strukturen-Akteure-Perspektiven", Bonn, 1985. Heribert Adam und Stanley Uys "Eight New Realities in Southern Africa" in CSIS Africa Notes (Washington) vom 28. Februar 1985, S. 1-8. Kenneth W. Grundy "Pax Pretoriana: South Africa's Regional Policy“ in Current History, 1985. April, S. 150-154. Klaus Frhr. vonder Ropp "Afrikas Süden im Zeichen der Pax Pretoriana" in Außenpolitik 1984. 4. Quartal, S. 419-433 im Anschluß an ders. "Frieden oder Krieg im südlichen Afrika?" in Außenpolitik 1977. 4. Quartal, S. 437-454.

4 Zur derzeitigen amerikanischen Politik im südlichen Afrika s. George Shultz in Southern African Record No. 35 (April 1984), S. 43-47; ders. in Wireless Bulletin from Washington vom 17. April 1985, S. 13-24; Lawrence S. Eagleburger in South Africa Foundation News von August 1983, S. 2-3, Chester A. Crocker in Wireless Bulletin from Washington vom 27. September 1984, S. 3-11 und Frank Wisner in South Africa International 1984. Januar, S. 467-475. 
Implementierung des von der westlichen Kontaktgruppe erarbeiteten, in Res. 435 des SR der Vereinten Nationen niedergelegten Planes und damit insbesondere auf der Abhaltung freier Wahlen. Südafrika fürchtete den Ausgang dieser Wahlen, die einen haushohen Sieg SWAPOs gebracht haben würden, und plädierte für die Einsetzung einer Regierung der nationalen Versöhnung in Windhoek. An ihr sollten außer SWAPO auch die sog. internen Parteien des Landes teilhaben. Damit wären Wahlen fürs erste obsolet gewesen. Als Pretoria mit seinen Vorstellungen nicht durchdrang, setzte es im Juni 1985 abermals eine Regierung der internen Parteien ein. Auch zu deren militärischem Schutz dringen inzwischen wieder Soldaten der South African Defence Forces (SADF) bei der Bekämpfung SWAPOs nach Angola ein.

Die Unterzeichnung (März 1984) des südafrikanisch-mozambiquanischen Anti-Subversions-Abkommens ${ }^{5}$ ("Vertrag von Nknomati“) erwies sich im Nachhinein als Fehlschlag. Mozambique verwies abredegemäß, und dies zum Entsetzen fast des gesamten schwarzen Afrikas, den (südafrikanischen) African National Congress (ANC) des Landes. Damit ging der ANC seiner wichtigsten Aufmarsch- und Rückzugsposition verlustig. Pretoria unterstützte dagegen, und dies im krassen Widerspruch zu seinen vertraglichen Verpflichtungen, weiterhin die mozambiquanische Widerstandsbewegung Renamo. Dies geschah durch eigene Leistungen und dadurch, daß Pretoria zuließ, daß sie aus den Kreisen der Hunderttausenden im Transvaal ansässiger "retornados" (d. s. die aus Angola und Mozambique vertriebenen portugiesischen Siedler) unterstützt wurden. Die "Friedensgespräche", zu denen sich im September/Oktober 1984 Vertreter der Regierung in Maputo und Renamos in Pretoria trafen, scheiterten. Zwar wurde dort die "Deklaration von Pretoria" verabschiedet, jedoch wurden die darin enthaltenen äußerst vagen Formulierungen zur Beendigung des Mozambique-Konfliktes nie konkretisiert. Insbesondere kam eine Koalitionsregierung Frelimo/Renamo nicht zustande. Wahrscheinlich hatte Renamo, übrigens eine Bewegung ohne klare Befehlsstrukturen und nicht frei von starken Elementen des reinen Banditentums, für die Verhandlungen in Pretoria gar kein Mandat gehabt. Abzuwarten bleibt, ob die jetzt in Mozambique eingesetzten tanzanianischen und zimbabwischen Truppen die Volksrepublik aus dem Zustand bewaffneter Anarchie herausführen können. Andernfalls droht totale Desintegration.

Wäre es der südafrikanischen Diplomatie gelungen, den Konflikt in Mozambique beizulegen, so hätte vielleicht eine Chance bestanden, auch für den angolanischen Bürgerkrieg eine politische Lösung zu finden. In Washington war man seit langem bemüht, Vertreter der früher auch von der OAU als solche anerkannten Befreiungsbewegung UNITA in die Regierung in Luanda aufzunehmen. Dies Bestreben erlitt einen Rückschlag, als der amerikanische Kongress im Sommer 1985 das "Clark Amendment" widerrief. Dieses 1976 von Senator Dick Clark initiierte Gesetz verbot Washington, angolanische Rebellenbewegungen (darunter als die weitaus wichtigste UNITA) zu unterstützen. Pretoria unternimmt weiter alles, um die dem ANC und SWAPO verbündete, ohnehin schwache

5 Zum Inhalt dieses Vertrages s. vor allem Winrich Kühne, a.a.O., S. 34-41. 
Regierung in Luanda weiter zu schwächen. Erwähnt sei hier nur die Entsendung eines südafrikanischen Kommandos nach Cabinda (Angola); es hatte den Auftrag, auf die dortigen, der amerikanischen Gulf Oil gehörenden Raffinerien einen Anschlag zu verüben. Wäre dies gelungen, so hätte Angola eine seiner ganz wenigen verbliebenen Möglichkeiten eingebüßt, harte Währungen zu verdienen.

Erfolgreicher gestaltete Pretoria seine Beziehungen zu dem schwachen Swaziland. Mit dem Königreich, so wurde 1984 bekannt, hatte Pretoria schon zwei Jahre zuvor einen Vertrag in der Art des Abkommens von Nknomati geschlossen. Bemühungen, auch mit Botswana und Lesotho zu solchen Ubereinkommen zu gelangen, scheiterten an deren Widerstand. Uberfälle der SADF auf Einrichtungen des ANC in Maseru und Gaborone wiesen beide Länder im Dezember 1982 bzw. im Sommer 1985 in ihre Schranken. Es heißt, daß Pretoria die Regierung in Harare/Zimbabwe dadurch einzuschüchtern wußte, daß es Parteigänger des inzwischen entmachteten Joshua Nkomo, die aus den Streitkräften des Landes dersertiert waren, weiter ausbildete und auch im übrigen unterstützte (sog. Super-ZAPUs).

Kein Staat in der Region ist auch nur annähernd in der Lage, der militärischen und wirtschaftlichen Vormacht an der Südspitze des afrikanischen Kontinents Paroli zu bieten. Gefahr droht Pretoria hingegen von Innen.

\section{Von der Unfähigkeit Pretorias zum friedlichen Wandel}

So stark die außen- und sicherheitspolitische Stellung Südafrikas nach alledem auch ist, so sehr unfähig bleibt die Regierung der weißen Afrikaner, zumindest ihre drängendsten internen Probleme zu lösen. Zwar wird in Pretoria, und dies nicht erst angesichts der gegenwärtigen Unruhen und der daraus resultierenden katastrophalen Entwicklungen für die Wirtschaft, durchaus gesehen, daß Apartheid gescheitert ist. Jedoch ist man unfähig, ${ }^{6}$ eine neue Politik zu formulieren.

\section{1) Modifizierungen im System der Apartheid}

Die allen, stets vagen, entgegenstehenden Äußerungen zum Trotz bis auf den heutigen Tag fortgeltende Politik der "Großen Apartheid" sieht vor, die zehn allesamt nicht lebensfähigen Reservate schwarzer Stämme ("Nationen") aus Südafrika anzugliedern und in die staatliche Unabhängigkeit zu entlassen. In den Fällen von vier dieser Reservate ist dies im vergangenen Jahrzehnt bereits geschehen. Sie bilden gemeinsam mit dem südafrikanischen Mutterland die in vielem noch zu entwickelnde "Konföderation von

6 Zu dem, was hier erforderlich ist s. Lawrence Schlemmer "African Political Rights: Part Two - Reform and Acceptability“ in Political Monitor/Indicator South Africa (Durban) 1985. Januar S. 1-6. 
Staaten des südlichen Afrikas". Gemeinsam mit etwa neun Anrainerstaaten soll sie die "Konstellation von Staaten des südlichen Afrikas" bilden.

Seit Anfang 1985 gilt in Südafrika eine neue Verfassung, die den gemischtrassigen und indienstämmigen Südafrikaner (d. s. zusammen ca. 12 v. H. einer Gesamtbevölkerung von etwa 32 Millionen) im Wege eines sog. Drei-Kammer-Systems ein sehr bescheidenes Mitspracherecht gewährt. Die bisherige Praxis zeigt, daß die Weißen (ca. 15 v. H.) weiterhin alleine herrschen. Seit Ende 1983 amtieren, soweit die jüngsten Unruhen sie nicht hinweggef egt haben, in über 100 (schwarzen) "townships" im "weißen" Südafrika autonome Stadträte. Aus deren Reihen sollen Delegierte in die neu zu schaffenden multirassischen "Regional Service Councils" entsandt werden und dort u. a. über Fragen der Regionalplanung mitentscheiden. Selbst ausgeprägt "verligte" Kreise in der regierenden (weißen) Nasionale Party van Suid-Afrika (NP) lehnen es ab, den im weißen Südafrika lebenden (ca. 11 Mio) schwarzen Südafrikanern eine verfassungsrechtlich ähnliche Position wie den Braunen zu geben: ". . Die reinfacher Lösung einer vierten Kammer des Parlaments ist rundum zurückgewiesen worden . . . die Strukturen werden sehr verschiedenen von denen sein, die innerhalb des Systems des Drei-Kammer-Parlaments bereits bestehen . . ."? Sähe Pretoria dies anders, so wäre doch nichts gewonnen. Denn eine solche Lösung stieße bei der erbärmlichsten Marionette auf nicht weniger Widerstand als bei den Anhängern der gefolgschaftsstarken Befreiungsbewegungen UDF/ANC, AZAPO/PAC und INKATHA. Es heißt, daß man in Pretoria dennoch erwägt, für die "städtischen" Schwarzen, parallel zu dem bestehenden weiß/braunen DreiKammer-System ein parlamentsähnliches Gremium zu schaffen. Auch mag daran gedacht sein, ein oder zwei schwarze Minister ohne Geschäftsbereich in das Kabinett sowie Schwarze in den sog. Präsidialrat aufzunehmen. Solche Uberlegungen können nur Ausdruck von Ratlosigkeit sein. Zu finanzieren sind übrigens schon die bestehenden Strukturen nicht mehr. ${ }^{8}$

\section{2) Stärken und Schwächen des schwarzen Protestes}

Vor allem 1960 und 1976/77 war Südafrika Schauplatz schwerer Unruhen. ${ }^{9}$ Auch damals sah eine häufig wenig informierte und entsprechend vorschnell urteilende Außenwelt die Regierung binnen kurzem stürzen und das Gros der Weißen, gleichgültig ob af rikaans-, englisch- oder portugiesischsprachig Südafrika fluchtartig verlassen. Dazu

7 C. J. van der Merwe "En wat van die Swartmense?«, S. 7, 8, hrsg. vom Federale Inligtingsdiens van die Nasionale Party, Kaapstad, April 1985.

8 Eine scharfe Kritik an der Ineffizienz und den Kosten der bestehenden Strukturen findet sich bei Frederik Van Zyl Slabbert, dem Führer der offiziellen Opposition, in Hansard (Kapstadt) vom 26. Juni 1984, Sp. 9827-9839.

9 Zu den Unruhen von 1960 finden sich Beiträge von Christopher Saunders, Oscar Wollheim und Alan Paton "Reflections on Sharpeville" in South African Outlook (Rondebosch) 1985. März, S. 35-37. S. ferner Graham Howe "Cycles of Civil Unrest" in Political Monitor/Indicator SA (Durban) Winter 1985, S. 7-12. 
wird es 1985 genauso wenig kommen! Allerdings ist unstreitig, daß die im August 1984 ausgebrochenen Unruhen alles zuvor Dagewesene in den Schatten stellen. Auch die seit Oktober 1984, so die offizielle Darstellung durch Minister Louis Le Grange, zur Niederschlagung der Aufstände eingesetzten SADF vermochten bislang "Recht und Ordnung" nicht wiederherzustellen. Und selbst konservative Politiker und Wirtschaftler Westeuropas und Nordamerikas beginnen zwischen sich und die "Friesen Afrikas" (Arnulf Baring) Distanz zu legen.

Getragen werden die Aufstände von der dem ANC nahestehenden United Democratic Front (UDF), ${ }^{10}$ einem im August 1983 ins Leben gerufenen Dachverband gesellschaftlicher Gruppen aller Art sowie dem nur wenige Monate älteren National Forum (NF)/AZAPO, das dem, (wie der ANC) seit über zwei Jahrzehnten verbotenen PAC nahesteht. Die multirassische UDF und die schwarzafrikanische (NF) AZAPO eint die kompromißlose Entschlossenheit, Apartheid mit allen zur Verfügung stehenden Mitteln zu bekämpfen und durch eine revolutionäre, "demokratische" Ordnung zu ersetzen. Beide trennt u. a. die Rivalität um die Herrschaft in der künftigen Volksrepublik Azania. ${ }^{11}$

Einen ersten Höhepunkt der jüngsten Welle des Protestes bildete im November 1984 eine Arbeitsniederlegung, an der südlich von Johannesburg 48 Stunden Hunderttausende von schwarzen Arbeitern und Angestellten teilnahmen. ${ }^{12}$ Nur wenige gemischtrassige und fast gar keine indieîstämmigen Afrikaner schlossen sich an. Sie streikten zusammen mit Schülern und Studenten, die bereits seit längerem den Unterricht boykottierten. Die Ziele des Streiks waren politischer Natur. Hier kam erstmals die gemeinsame Mitgliedschaft in der UDF zum Tragen. In den folgenden Monaten stand das Bestreben von UDF und AZAPO im Vordergrund, möglichst viele Städte unregierbar zu machen. ${ }^{13}$ Dazu gehörte vor allem der Kampf gegen schwarze Kollaborateure, d. w. insbesondere Polizisten und Mitglieder der Stadträte. Ihnen boten sich oft nur zwei Optionen: der Rückzug aus dem Amt oder die Flucht in die Polizeikasernen oder sonstige Gebäude der Weißen. Wer beides nicht tat, der lief Gefahr, inmitten einer johlenden Menge, vielleicht mitsamt seiner Familie, gerichtet und verurteilt zu werden, "mob execution by burning." Entsprechende Filme von Menschen, die bei lebendigem Leib verbrannt wurden, wurden auch im Ausland gezeigt und verfehlten dort ihre Wirkung nicht. Eine perfide Propaganda war bemüht den Eindruck zu erwecken, als billigten führende Geistliche aus den Kreisen des oppositionellen SA Council of Churches (SACC), darunter der von der Bekennenden Kirche geprägte bedeutende Theologe und Rebell Christian Frederik

10 Zur UDF s. J. Hennie P. Serfontein "The United Democratic Front the most significant national movement since the sixties?" in Ecunews (Johannesburg) 1983. August, s. 12, 14 S. weiter "The United Democratic Front/Another bubble, or is this one for real?" in Frontline (Johannesburg) 1983. Oktober, S. 42-47.

11 Dazu Beatrice Hollyer "Targets of Contrast" in Frontline (Johannesburg) 1985. April, S. 8-12.

12 Dazu Graham Howe "The Stayaway Strikes of 1984" in Urban Monitor/Indicator SA (Durban) 1985. Januar, S. 1-5.

13 Dazu "A Chronology of Township Unrest/6 Months of Dissent - 1984" in Urban Monitor/Indicator SA (Durban) 1985. Januar, S. 6-9. 
Beyers Naudé, dies Vorgehen der jungen Revolutionäre. Viele von ihnen befanden sich übrigens noch im Kindesalter. Polizei und Armee standen, was die Brutalität des Vorgehens anbelangt, den Aufbegehrenden in nichts nach.

Derzeit läßt sich noch schwer beurteilen, ob es den Behörden durch die Verhängung des Ausnahmezustandes in 36 Gerichtsbezirken im Juli 1985 gelungen ist, die Lage entscheidend zu beruhigen. Auch die Zensur erschwert hier die Urteilsfindung.

Die Aufstände, die sich auf den östlichen Rand, das östliche Kap und zeitweise auch die Umgebung von Durban konzentrieren, boten gute Gelegenheit zur Begleichung von alten Rechnungen. Zwischen Anhängern von UDF/ANC und INKATHA; vereinzelt auch zwischen Anhängern der AZAPO/PAC und INKATHA; zwischen Zulus und "Indern"; Auseinandersetzungen tribalistischer Natur usw. Auch nutzten viele die Gunst der Stunde, herkömmliche Raubüberfälle, Einbrüche, Morde u. ä. zu begehen. Oft hatte es den Anschein, als interveniere die Polizei nicht, obgleich ihr das möglich war. Der Terror der Revolutionäre und die allgemeine Gesetzlosigkeit würde auf Dauer dem Befreiungskampf der Schwarzen eher schaden als nützen.

Auch andere Waffen machten den Weißen schwer zu schaffen. Zu nennen ist hier etwa der Boykott weißer Geschäfte durch schwarze Kunden. Wer von den letzteren den entsprechenden Aufrufen nicht folgte, der wurde von den Revolutionären mit brachialer Gewalt zur Beachtung ihrer Befehle gezwungen. So wurde etwa der Beruf des Fahrers eines Lieferantenwagens eines weißen Kaufhauses zu einem der gefährlichsten Berufe. Trotz alledem sollte eines nicht übersehen werden: Südafrika ist bislang bei weitem nicht das "Land in Flammen", als das es westliche Berichterstatter oft ausgeben. So läuft etwa die Produktion in diesem hochindustrialisierten Land trotz der Störanfälligkeit seiner Volkswirtschaft weiter. Es gibt bislang nur ganz vereinzelte Ubergriffe auf weiße Wohngebiete. Umkhonto we Sizwe, die Guerillaarmee des ANC, hat bislang kaum "soft targets" attackiert. Die "Kriegserklärung", die der ANC im Juni 1985 in Kabwe bei seiner "National Consultative Conference" an Pretoria richtete, machte aber deutlich, in welcher Richtung der ANC künftig operieren wird. ${ }^{14}$

Erst die Aufteilung des "weißen" Südafrikas in braune, schwarze und weiße Wohngebiete macht es möglich, daß die Bewohner weißer Städte häufig nur eine sehr vage Ahnung von den revolutionären Entwicklungen in den schwarzen Städten haben. Die schärfer gewordene Zensur tut ein übriges, so daß sich immer noch viele Weiße in falscher Sicherheit wiegen. Die führenden Kreise der Wirtschaft sind besser informiert.

14 S. in diesem Zusammenhang schon Andrew Prior "The ANC and the stalled revolution" in Frontline (Johannesburg) 1985. März, S. 6-7.

15 Sehr informativ Robert von Lucius "Die Kampagne gegen Investitionen in Südafrika kann auch in Europa immer mehr 'Erfolger verzeichnen“ in FAZ vom 3. Juli 1985, S. 15. 


\section{3) Reaktionen der Wirtschaft}

Der Ernst der Lage ist wirtschaftlichen Entwicklungen zu entnehmen. Der Verfall des Randes macht dies deutlich. Mitte September 1985 wurde er nur noch mit 38 US Cents gehandelt, obwohl er unter Zugrundelegung rein wirtschaftlicher Kriterien 50 US Cents hätte kosten müssen. Und selbst ein Kurs von 50 US Cents wäre Ausdruck des Verfalls seines externen Wertes in den zurückliegenden 18 Monaten gewesen.

Heute sind in- und ausländische Wirtschaftskreise wohl häufig der Meinung, daß die innenpolitischen Probleme Südafrikas mit friedlichen Mitteln kaum lösbar sind, zumindest aber die Regierung Pieter Willem Bothas dazu nicht in der Lage ist. Dem südafrikanischen Regierungschef fehlt alles, was Charles de Gaulle seinerzeit zur Lösung des Algerienproblems befähigte. Zwar machen südafrikanische Wirtschaftskreise heute ihre Unzufriedenheit mit Bothas "Reformkurs" deutlich, jedoch sind sie letztlich genauso ratlos wie die Regierung. Auch sie wissen nicht, wie ein Ausgleich zwischen den imkompatiblen Forderungen des schwarzen und des weißen Nationalismus friedlich zu erzielen ist. Sehr bemerkenswert ist, daß sich im September 1985 eine sehr kleine Gruppe führender Industrieller und Journalisten in Zambia zu Gesprächen mit den Verantwortlichen des ANC aufhielt.

Anders als früher finden die Aufstände 1985 vor dem Hintergrund hoher Arbeitslosigkeit, hoher Inflationsrate und der anderer Merkmale einer schweren Wirtschaftskrise statt. Die Lage wurde durch die im Spätsommer aufgetretene Liquiditätskrise verschärft. Hier spielte die teils legale, teils illegale Flucht in- und ausländischen Kapitals eine wichtige Rolle. Wichtiger war vielleicht noch, daß sich ausländische Geschäftsbanken jetzt weigerten, Umschuldungsabkommen über kurzfristige Forderungen zu schließen. Früher war dies routinemäßig geschehen. Aus alledem resultierte der Kursverfall. Die zuständigen Stellen sahen sich Ende August genötigt, Devisengeschäfte und Aktienhandel für einige Tage einzustellen. Das Unvermögen, Zusagen für fundamentalen politischen Wandel zu machen, ließ die Reise des Chefs der südafrikanischen Notenbank in Länder der westlichen Welt zu einer Odyssee werden. Nachdem infolge von Druck engagierter Apartheid-Gegner US-Banken schon seit Jahren zögerlich waren, mit Südafrikaner Geschäfte abzuschließen, stieß Gerhard de Kock auch in Westeuropa auf taube Ohren. Die Antwort Pretorias war ein selbstverkündetes, bis Ende 1985 befristetes Auslandsschulden-Moratorium. Namentlich amerikanische Experten sahen darin einen "Kinnhaken" für die künftige Kreditwürdigkeit Südafrikas.

\section{Neue Elemente in der Südafrika-Politik westlicher Staaten}

Das vielleicht nicht erst mittel- bis längerfristig von Bürgerkrieg und Revolution bedrohte Südafrika hat viel von seiner einstigen Attraktivität als Wirtschaftspartner eingebüßt. Das hat dazu beigetragen, daß jene Kreise, die seit eh und je der Verhängung von Sanktionen gegen Pretoria das Wort reden, heute mehr Erfolge verbuchen können als 
jemals zuvor. Besonders auffällig ist das in den USA. 1985 setzen hier Republikaner Maßnahmen durch, die selbst zur Zeit Jimmy Carters kaum denkbar waren.

Südafrika befürchtet eine langsame aber stetige Eskalation von wirtschaftlichen Sanktionen und Zwangsmaßnahmen anderer Art. Insofern sind auch die Maßnahmen wichtig, die Australien, Kanada und andere Länder gegen Pretoria ergriffen haben, die allesamt wenig Wirtschaftsbeziehungen zu Südafrika unterhalten. ${ }^{15}$ Wichtiger ist die Rückberufung des französischen Botschafters und das Verbot neuer französischer Investitionen in Südafrika. Wirklich schwerwiegend sind Maßnahmen, die Japan, die USA, Großbritannien und die BR Deutschland, die mit Abstand wichtigsten Handelspartner der Republik am Kap, ergreifen.

In den USA ist die Politik des "constructive engagements" heute noch umstrittener als zuvor. Ähnlich wie in der Bundesrepublik, so hat sich auch in den USA ein Mythos entwickelt, als habe die in der zweiten Hälfte der siebziger Jahre verfolgte Politik eine wirkliche Erfolgschance gehabt. Häufig wird in den USA dem Desinvestment, dem Rückzug der amerikanischen Investitionen, das Wort geredet. Es wird Druck auf Unternehmen ausgeübt, die Kontakte nach Südafrika unterhalten. Universitäten, Kirchen, Pensionsfonds und andere Institutionen werden gedrängt, ihr Kapital nicht mehr in Aktien solcher Unternehmen anzulegen, die Südafrika geschäftlich verbunden sind. In Washington, New York und Atlanta reißen die Anti-Apartheid Demonstrationen nicht mehr ab. So veranstaltet etwa das "Free South Africa Movement" seit über einem Jahr vor der südafrikanischen Botschaft in Washington illegale Demonstrationen. Vor diesem Hintergrund wird verständlich, daß beide Häuser des Kongresses mit den Stimmen von Demokraten und Republikanern und damit mit überwältigenden Mehrheiten Gesetzesvorlagen verabschiedeten, die fühlbare Wirtschaftssanktionen enthielten. Zunächst wurde damit gerechnet, daß R. Reagan gegen ein entsprechendes Gesetz sein Veto einlegen würde. Es waren dann vor allem führende Republikaner, die den Präsidenten warnten, sein Veto werde vom Kongreß mit Zwei-Drittel-Mehrheit überstimmt werden. So blieb Reagan, wollte er nicht in einer innenpolitisch höchst sensitiven Frage eine Niederlage in Kauf nehmen, keine andere Wahl, als selbst Sanktionen gegen Südafrika zu verhängen. Diese fielen erwartungsgemäß milde aus. Sie umfassen vor allem die Ausfuhr von Computern an staatliche Stellen, den Export von Installationen für die Nuklearindustrie und Atom-Technologie sowie Kredite zugunsten staatlicher Stellen alle derartigen Geschäfte werden untersagt. ${ }^{16}$ Abzuwarten bleibt, ob sich der Kongreß mit diesen Maßnahmen zufriedengibt oder härtere Sanktionen gegen das dann wohl zu erwartende Veto des Präsidenten durchsetzt.

Die Entwicklungen in den USA wie auch die Verhängung des Notstandes in Regionen Südafrikas brachten die EG und ihre Mitglieder dahin, ihre bisherige Position neu zu überdenken. Dabei mag eine Rolle gespielt haben, daß es zwei EG-Staaten (Dänemark und Frankreich) waren, die im Juli 1985 erfolgreich eine Empfehlung des Sicherheitsra-

16 Dazu "Reagan Orders Measures Against South Africa" in Wireless Bulletin from Washington (Bonn) vom 10. September 1985, S. 1-4. 
tes der Vereinten Nationen (Res. 569) initiiert hatten, wonach Investitionen in Südafrika zu suspendieren, der Verkauf von in Südafrika geprägten Goldmünzen zu verbieten, Exportrisikogarantien auszusetzen und der Verkauf von Nuklearanlagen und -geräten sowie solcher Elektronik, die der Armee und Sicherheitspolizei dienen können, zu untersagen seien. ${ }^{17}$ Hingegen vertraten Luxemburg, die BR Deutschland und Großbritannien die Auffassung, daß Sanktionen eher schädlich und von daher von ihrer Verhängung abzusehen sei. Hier wird die Warnung von Herbst 1978 fortwirken, als Vertretern westlicher Staaten in Pretoria bedeutet wurde, daß sie im Bereich der Versorgung mit strategischen Rohstoffen das Opfer von Gegensanktionen werden könnten. Auch wird niemand ausschließen, daß Pretoria als Gegenmaßnahmen einen Teil oder alle jener ca. 350000 schwarzen Arbeiter, die aus den angrenzenden Nachbarstaaten zur Arbeitsaufnahme nach Südafrika gekommen sind, in ihre Ursprungsländer abschieben könnte. Dort würden sie die ohnehin vorhandene wirtschaftliche Misere noch vergrößern.

Ende August 1985 unternahmen die Außenminister Luxemburgs, der Niederlande und Italiens gemeinsam mit dem für auswärtige Angelegenheiten zuständigen EG-Kommissar eine Erkundungsmission, die sie für drei (!) Tage nach Südafrika führte. Wichtiger war wahrscheinlich die Unterredung, zu der, gleichfalls namens der EG, der luxemburgische Präsident des EG-Ministerrates kurze Zeit später eine ANC-Delegation empfing. Im September 1985 entschieden sich die EG-Staaten (incl. Spanien und Portugal) dafür, die Lieferung militärischer Güter noch weiter einzuschränken und keine neuen Verträge im Nuklearsektor abzuschließen. Ferner wurde eine Einschränkung bei Erdöltransporten beschlossen. Soweit solche überhaupt noch bestehen, sollen auch sportliche und kulturelle Kontakte abgebaut werden. Schließlich werden die EG-Staaten, an deren Botschaften in Pretoria/Kapstadt Militärattachés Dienst tun, diese abziehen.

So unbefriedigend diese Maßnahmen in den Augen des schwarzen Südafrikas auch sein mögen, so mag, und das wird in Pretoria ganz realistisch gesehen, von ihnen doch Präzedenzwirkung ausgehen. Südafrika ist dem Status eines Paria-Staates im Sommer 1985 um etliches näher gekommen.

Anders als früher, so ist man heute in den westlichen Staaten wohl weitgehend einig, daß Sanktionen zum Ziel haben müssen, die Regierung in Pretoria zu veranlassen, mit den Vertretern von UDF/ANC, INKATHA, PAC/AZAPO und weiteren Gruppen, darunter vor allem Gewerkschafts- und Kirchenvertretern, über eine neue politische Ordnung für Südafrika zu verhandeln. Erstaunlich ist, daß sich, sieht man von London und Washington ab, die Regierungen, die Südafrika zu solchen Verhandlungen drängen, selbst allem Anschein nach kaum Gedanken über die Frage gemacht haben, wie eine solche neue Ordnung aussehen könnte. So bemerkt etwa der weißafrikanische Humboldt-Stipendiat Deon Geldenhuys am Ende seiner vorzüglichen Studie über die einschlägige Diskussion in der Bundesrepublik "... Uberrascht ist der südafrikanische Beobachter zu erfahren, daß die mit soviel Expertise, Leidenschaft und Offenheit ge-

17 Dazu vor allem "Folgen einer Entschließung des UNO-Sicherheitsrates/Die Ächtung Südafrikas bei den internationalen Wirtschaftsorganisationen" in NZZ vom 9. August 1985, S. 8. 
führte deutsche Diskussion in der konkreten Politik der Bundesrepublik nie Berücksichtigung gefunden hat. Sie wird hier ignoriert! ${ }^{18}$ Und das obwohl aus der Zeit der Kanzlerschaft Helmut Schmidts folgende Begebenheit berichtet wird: Nach einem Treffen mit dem damaligen Ministerpräsidenten J. B. Vorster im Mai 1977 in Wien äußerte der seinerzeitige amerikanische Vize-Präsident Walter Mondale gegenüber H. Schmidt "We must put all kinds of pressure on SA, so that it gives up Apartheid"; eine Forderung, die Schmidt um das lakonische "and replace it by what?" ergänzte. Dem früheren Bundeskanzler war klar, daß, um dies mit den Worten des Theologen Helmut Gollwitzer zu sagen, ${ }^{19}$ die Befreiung der weißen Minderheit in Südafrika von ihrer (Existenz-)Angst ein psychologisch zentrales Problem ist. Schlüssel für die Befreiung des schwarzen Südafrikas wird noch lange die Sicherheit des weißen Südafrikas sein! Hier ist nicht der Raum darzustellen, durch welche Formen staatlicher Machtteilung ${ }^{20}$ oder einer geographischen Teilung des Landes oder eine Kombination beider Vorstellungen dies Postulat zu verwirklichen ist. Dies ist jedoch unschädlich, da Deon Geldenhuys in seiner bereits erwähnten Studie alle wichtigen Úberlegungen aufgezeigt hat.

18 "Die Zukunft Südafrikas aus deutscher Sicht" in Außenpolitik 1985. 1. Quartal, S. 80-98.

19 In einem Interview "Jesus ist heute kein Weißer" in Die Zeit vom 1. Juni 1984, S. 56.

20 Dazu aus der Sicht INKATHAs "Facing up to Sharing Power" in Clarion Call (Ulundi) 1985, S. 5-13. 
self-interested motives is one of the reasons for a widening gap between the German public's practical formulation of government policy.

The officially proclaimed goal of strengthening the dynamic forces in the developing countries by giving aid for supporting self-help efforts is criticized for two reasons: first, about two thirds of of ficial development aid is given to the governments of developing countries, and it is their behaviour which strongly influences the developmental effects of the aid; second, economic development contraints have been overstated at the expense of socio-political constraints. This view has led to two proposals for future aid strategy: one is to give aid only where the donor is absolutely certain that it will be used for elementary needs in a really poor country; the other is to strengthen so-called "social-technologytransfer". The latter strategy demands a climate of policy dialogue which gives confidence to the governments and the populations of developing countries to make use of aid. A development aid policy which is dominated by shortterm political and economic self-interest tends to be counter-productive to such a policy dialogue and prevents efforts to look for new solutions to overcome the present development crisis of the countries of the Third World.

\section{South Africa - Prepotency and Impotence}

\section{By Klaus Frhr. von der Ropp}

As is well known, the administration of former U.S. president Jimmy Carter was deeply involved in trying to overcome Apartheid; it naively assumed that it could help to introduce the "American model" at the Cape of Good Hope. The Reagan administration, on the other hand, is prepared to admit that Apartheid, because of South Africa's uniquely heterogeneous population, must be replaced by a new order sui generis. Both administrations failed, as Pretoria is not prepared to negotiate with the true representatives of Black South Africa.

South Africa dominates developments in most parts of Southern Africa more or less with ease. But Pretoria is totally unable to solve its domestic problems. Consequently there have been, since August 1984, more serious uprisings in many parts of the country than ever before. There is open anarchy in many Black townships. In many of them the often very young revolutionaries have succeeded in making them ungovernable. Even if many leading white South Africans today realise that Apartheid was an error, they do not know by which new order to replace it. The future holds for South Africa a very bloody civil war. This is clearly recognised by Western and other economic interest groups; they realise that for a long time South Africa will have lost its attractiveness as an economic partner. Today American Republicans impose sanctions against South Africa that American Democrats had hardly thought of only a few years ago. But it still holds true that any solution which is to bring liberation to South Africa must include copper-bottomed guarantees of secure existence for Whites. 